\title{
A QUADRATURE FORMULA INVOLVING ZEROS OF BESSEL FUNCTIONS
}

\author{
CLÉMENT FRAPPIER AND PATRICK OLIVIER
}

\begin{abstract}
An exact quadrature formula for entire functions of exponential type is obtained. The nodes of the formula are zeros of the Bessel function of the first kind of order $\alpha$. It generalizes and refines a known quadrature formula related to the sampling theorem. The uniqueness of the nodes is studied.
\end{abstract}

\section{INTRODUCTION}

Given any polynomial $p$ of degree $<n$ and $n$ distinct numbers $x_{1}, x_{2}$, $\ldots, x_{n}$, the classical Lagrange interpolation formula is

$$
p(x)=\sum_{k=1}^{n} p\left(x_{k}\right) \frac{l(x)}{\left(x-x_{k}\right) l^{\prime}\left(x_{k}\right)},
$$

where $l(x)=\prod_{j=1}^{n}\left(x-x_{j}\right)$. Multiplying both members of (1) by a weight function $w(x)$ and integrating, we obtain the quadrature formula

$$
\int_{-1}^{1} w(x) p(x) d x=\sum_{k=1}^{n} \lambda_{k} p\left(x_{k}\right)
$$

where

$$
\lambda_{k}=\int_{-1}^{1} w(x) \frac{l(x)}{\left(x-x_{k}\right) l^{\prime}\left(x_{k}\right)} d x .
$$

In order to obtain a quadrature formula valid for all polynomials of degree $<2 n$, we consider the Hermite interpolation formula

$$
p(x)=\sum_{k=1}^{n}\left(p\left(x_{k}\right) l_{k, 0}(x)+p^{\prime}\left(x_{k}\right) l_{k, 1}(x)\right),
$$

with

$$
l_{k, 0}(x)=\left(1-\frac{l^{\prime \prime}\left(x_{k}\right)}{l^{\prime}\left(x_{k}\right)}\left(x-x_{k}\right)\right)\left(\frac{l(x)}{\left(x-x_{k}\right) l^{\prime}\left(x_{k}\right)}\right)^{2}
$$

and

Received by the editor November 12, 1991.

1991 Mathematics Subject Classification. Primary 41A55, 33C10, 41 A05.

Key words and phrases. Quadrature formula, nodes, entire functions, Bessel functions.

This research was supported by the Natural Sciences and Engineering Research Council of Canada Grant No. OGP0009331. 


$$
l_{k, 1}(x)=\left(x-x_{k}\right)\left(\frac{l(x)}{\left(x-x_{k}\right) l^{\prime}\left(x_{k}\right)}\right)^{2} \text {. }
$$

This time we obtain

$$
\int_{-1}^{1} w(x) p(x)=\sum_{k=1}^{n}\left(\lambda_{k, 0} p\left(x_{k}\right)+\lambda_{k, 1} p^{\prime}\left(x_{k}\right)\right),
$$

where

$$
\lambda_{k, r}=\int_{-1}^{1} w(x) l_{k, r}(x) d x .
$$

If we take, in particular, the $n$th orthogonal polynomial $l(x)=Q_{n}(x)$ associated with the weight function $w(x)$, then [6] we have $\lambda_{k, 1}=0$ and $\lambda_{k, 0}=\lambda_{k}$, $1 \leq k \leq n$. Therefore, the quadrature formula (2) is valid for all polynomials of degree $<2 n$. Moreover, the zeros of $Q_{n}(x)$ are the only points having this property.

The Jacobi polynomial of degree $n, Q_{n}(x)=P_{n}^{(\alpha, \beta)}(x)$, is, with $w(x)=$ $(1-x)^{\alpha}(1+x)^{\beta}$, an important special case . For the Chebyshev polynomial of the first kind of degree $n$,

$$
T_{n}(x)=\frac{2^{2 n}(n !)^{2}}{(2 n) !} P_{n}^{(-1 / 2,-1 / 2)}(x)=\cos (n \arccos x),
$$

we get the Gauss-Jacobi type formula

$$
\int_{-1}^{1} \frac{p(x)}{\sqrt{1-x^{2}}} d x=\frac{\pi}{n} \sum_{k=1}^{n} p\left(\cos \frac{(2 k-1) \pi}{2 n}\right) .
$$

This formula has been extended to entire functions. Let $B_{\sigma}$ be the class of entire functions of exponential type $\sigma$, bounded on the real axis. If $f \in B_{2 \tau}$ satisfies $f(x)=O\left(|x|^{-\delta}\right), \delta>1$, then [1]

$$
\int_{-\infty}^{\infty} f(x) d x=\frac{\pi}{\tau} \sum_{k=-\infty}^{\infty} f\left(\frac{(2 k-1) \pi}{2 \tau}\right) .
$$

This latter is in fact valid under weaker integrability conditions [2]. Here the nodes $(2 k-1) \pi /(2 \tau)$, the zeros of $\cos \tau z$, are related to those of formula (5) by

$$
\lim _{n \rightarrow \infty} \sqrt{\pi} \frac{P_{n}^{(-1 / 2,-1 / 2)}(\cos (\tau z / n))}{n^{-1 / 2}}=\cos \tau z .
$$

In general [6, p.167],

$$
\lim _{n \rightarrow \infty} \frac{P_{n}^{(\alpha, \beta)}(\cos (\tau z / n))}{n^{\alpha}}=\left(\frac{2}{\tau z}\right)^{\alpha} J_{\alpha}(\tau z),
$$

where $J_{\alpha}(z)$ is the Bessel function of the first kind of order $\alpha$. Thus, the nodes in (6) are the zeros of $J_{-1 / 2}(\tau z) /(\tau z)^{-1 / 2}$; it appears natural to generalize (6) using as nodes the zeros of $J_{\alpha}(\tau z) /(\tau z)^{\alpha}$. 
For the various properties of Bessel functions used in this paper, we refer the reader to [7].

\section{Statement of the Results}

The function

$$
\frac{J_{\alpha}(\tau z)}{(\tau z)^{\alpha}}=\sum_{k=0}^{\infty}(-1)^{k} \frac{(\tau z)^{2 k}}{2^{\alpha+2 k} k ! \Gamma(k+\alpha+1)}
$$

is an even entire function of exponential type $\tau$. Let $j_{k}=j_{k}(\alpha), k= \pm 1$, $\pm 2, \ldots$, be the zeros of $J_{\alpha}(z) / z^{\alpha}$ ordered such that $j_{-k}=-j_{k}$ and $0<\left|j_{1}\right| \leq$ $\left|j_{2}\right| \leq \ldots$. We are now ready to state our main result.

Theorem 1. Let $\Re(\alpha)>-1$. For all $f \in B_{2 \tau}$ such that $f(x)=O\left(|x|^{-\delta}\right), x \rightarrow$ $\pm \infty$, with $\delta>2 \Re(\alpha)+2$, we have

$$
\begin{aligned}
\int_{0}^{\infty} & x^{2 \alpha+1}(f(x)+f(-x)) d x \\
& =\frac{2}{\tau^{2 \alpha+2}} \sum_{k=1}^{\infty} \frac{j_{k}^{2 \alpha}}{\left(J_{\alpha}^{\prime}\left(j_{k}\right)\right)^{2}}\left(f\left(\frac{j_{k}}{\tau}\right)+f\left(-\frac{j_{k}}{\tau}\right)\right) .
\end{aligned}
$$

The particular case $\alpha=-1 / 2$ leads us to formula (6). Indeed, we have

$$
\frac{J_{-1 / 2}(\tau z)}{(\tau z)^{-1 / 2}}=\sqrt{\frac{2}{\pi}} \cos \tau z \quad \text { and } \quad j_{k}(-1 / 2)=(2 k-1) \frac{\pi}{2} \text {. }
$$

When $\alpha=1 / 2$ we have

$$
\frac{J_{1 / 2}(\tau z)}{(\tau z)^{1 / 2}}=\sqrt{\frac{2}{\pi}} \frac{\sin \tau z}{\tau z}
$$

and the formula (8) becomes

$$
\int_{-\infty}^{\infty} x^{2} f(x) d x=\frac{\pi}{\tau} \sum_{\substack{k=-\infty \\ k \neq 0}}^{\infty}\left(\frac{k \pi}{\tau}\right)^{2} f\left(\frac{k \pi}{\tau}\right),
$$

where $f(x)=O\left(|x|^{-\delta}\right), x \rightarrow \pm \infty, \delta>3$. Replacing $f(x)$ by

$$
\frac{1}{x^{2}}\left(f(x)-f(0)\left(\frac{\sin \tau x}{\tau x}\right)^{2}\right)
$$

where $f(x)$ can be supposed to be even, we obtain

$$
\int_{-\infty}^{\infty} f(x) d x=\frac{\pi}{\tau} \sum_{k=-\infty}^{\infty} f\left(\frac{k \pi}{\tau}\right)
$$

where $f(x)=O\left(|x|^{-\delta}\right), x \rightarrow \pm \infty, \delta>1$. Up to a translation, this formula is equivalent to $(6)$.

By considering a function of the form

$$
f(z)=F(z) \frac{J_{\alpha}(\tau z)}{z^{\alpha}\left(\tau z-j_{m}\right)},
$$

we obtain the following corollary of Theorem 1 . 
Corollary 1. Let $\Re(\alpha)>-1$. For all $F \in B_{\tau}$ such that $F(x)=O\left(|x|^{-\delta}\right), x \rightarrow$ $\pm \infty$, with $\delta>\Re(\alpha)+1 / 2$, we have

$$
F\left(\frac{j_{m}}{\tau}\right)=\frac{\tau^{\alpha+2}}{2 j_{m}^{\alpha}} J_{\alpha}^{\prime}\left(j_{m}\right) \int_{0}^{\infty} x^{\alpha+1} J_{\alpha}(\tau x)\left(\frac{F(x)}{\tau x-j_{m}}-\frac{F(-x)}{\tau x+j_{m}}\right) d x .
$$

Taking in (11) the function

$$
F(z)=\frac{J_{\alpha}(\tau z)}{z^{\alpha}\left(\tau z-j_{l}\right)},
$$

we deduce the following property of Bessel functions.

Corollary 2. Let $\Re(\alpha)>-1$. If $m$ and l are integers, then

$$
\begin{aligned}
& \frac{1}{2} \int_{0}^{\infty} x J_{\alpha}^{2}(x)\left(\frac{1}{\left(x-j_{m}\right)\left(x-j_{l}\right)}+\frac{1}{\left(x+j_{m}\right)\left(x+j_{l}\right)}\right) d x \\
& \quad= \begin{cases}0, & l \neq m, \\
1, & l=m .\end{cases}
\end{aligned}
$$

Note that the case $l=m$ of (12) will be used in the proof of Theorem 1 . So, we shall need an independent proof (see Lemma 4).

When $\alpha=1 / 2$, in Corollary 2 , we readily obtain the orthogonality property [4] of the family

$$
\left\{\frac{\sin \pi(x-m)}{\pi(x-m)}: m \in \mathbb{Z}\right\}
$$

namely,

$$
\int_{-\infty}^{\infty} \frac{\sin \pi(x-m)}{\pi(x-m)} \frac{\sin \pi(x-l)}{\pi(x-l)} d x= \begin{cases}0, & l \neq m \\ 1, & l=m .\end{cases}
$$

It has been proved [5] that formula (10) is the unique quadrature formula of the form

$$
\int_{-\infty}^{\infty} f(x) d x=\sum_{k=-\infty}^{\infty} \lambda_{k} f\left(x_{k}\right),
$$

which is valid for all $f \in B_{2 \tau}$. The nodes $x_{k}$ in (14) are completely characterized by an extremal problem: they are the roots of the function $\sin \tau x$, which minimizes the integral

$$
\int_{-\infty}^{\infty}\left(\frac{s(x)}{x}\right)^{2} d x
$$

over a certain subclass of $B_{\tau}$. The following theorem is the corresponding $(\alpha=-1 / 2)$ uniqueness result for quadrature formulas of the form

$$
\int_{0}^{\infty} x^{2 \alpha+1}(f(x)+f(-x)) d x=\sum_{k=-\infty}^{\infty} \lambda_{k} f\left(x_{k}\right) .
$$

Observe that if $\alpha$ is not real then the uniqueness does not hold. The formula (8), when $\alpha$ is replaced by $\bar{\alpha}$, is still valid for the same class of functions. To see this we need only to replace $f(z)$ by $\overline{f(\bar{z})}$ and to observe that $\overline{j_{k}(\alpha)}=j_{k}(\bar{\alpha})$. Therefore, we assume that the sequence $x_{k},-\infty<k<\infty$, in (15), is a sequence of distinct real numbers without an accumulation point in $\mathbb{R}$. We 
assume also that one of the nodes is a zero of $J_{\alpha}(\tau z)$. We associate, with every quadrature formula of the form (15) and every zero $j_{m}$ of $J_{\alpha}(z)$ (which has only real zeros for $\alpha>-1$ ), a class of entire functions $\omega$ with the following properties:

(i) $\omega \in B_{\tau}$,

(ii) $\omega(x) \in \mathbb{R}$ for $x \in \mathbb{R}$,

(iii) $\omega(x)=O\left(|x|^{-\alpha-3 / 2}\right), x \rightarrow \pm \infty$,

(iv) $\omega\left(x_{k}\right)=0, k \neq m$,

(v) $\omega\left(j_{m} / \tau\right)=1$.

Here the nodes $x_{k},-\infty<k<\infty$, have been ordered such that $x_{m}=j_{m} / \tau$. We call every such function $\omega$ a nodal function.

Theorem 2. Let $\alpha>-1$. Among all the quadrature formulas of the form (15) having $\omega(x)$ as nodal function, only one is valid for all $f \in B_{2 \tau}$ satisfying $f(x)=O\left(|x|^{-\delta}\right), x \rightarrow \pm \infty, \delta>2 \alpha+2$. This formula is (8), and the associated nodal function is

$$
\omega_{e}(x):=\frac{j_{m}^{\alpha}}{J_{\alpha}^{\prime}\left(j_{m}\right)} \frac{J_{\alpha}(\tau x)}{x^{\alpha}\left(\tau x-j_{m}\right)} .
$$

Moreover, $\omega_{e}$ minimizes the integral

$$
\int_{0}^{\infty} x^{2 \alpha+1}\left(\omega^{2}(x)+\omega^{2}(-x)\right) d x
$$

over all nodal functions $\omega$.

\section{AUXILIARY RESULTS}

For every entire function of exponential type satisfying certain conditions at infinity, we have the classical sampling theorem, namely

$$
f(x)=\sum_{k=-\infty}^{\infty}(-1)^{k} f\left(\frac{k \pi}{\tau}\right) \frac{\sin \tau x}{\tau x-k \pi} .
$$

Integrating formally both members of (16), we obtain formula (10) but only for $f \in B_{\tau}$. In order to prove a result valid for $f \in B_{2 \tau}$, we use an extension, to entire functions of exponential type, of formula (3). This extension (see Lemma 3 , below) is a generalization and a refinement of (16).

From now on we may suppose $\tau=1$. We need first a technical result.

Lemma 1. Let $z=R e^{i \theta}$ be a complex number on the circle $|z|=R:=N \pi+$ $\Re(\alpha) \pi / 2+\pi / 4$, where $N$ is a large positive integer. There exists a positive constant $C(\alpha)$ such that

$$
\left|J_{\alpha}(z)\right|>\frac{C(\alpha)}{\sqrt{R}} e^{R|\sin \theta|}, \quad|\theta| \leq \pi .
$$

Proof. Obviously, we may suppose $\Re(z) \geq 0$. In view of the asymptotic expansion

$$
J_{\alpha}(z)=\sqrt{\frac{2}{\pi z}} \cos \left(z-\alpha \frac{\pi}{2}-\frac{\pi}{4}\right)\left(1+O\left(|z|^{-1}\right)\right),
$$

it suffices to prove that

$$
\left|\cos \left(z-\alpha \frac{\pi}{2}-\frac{\pi}{4}\right)\right|>K(\alpha) e^{R|\sin \theta|} .
$$


Let $x=R \cos \theta, y=R \sin \theta, u=x-\Re(\alpha) \pi / 2-\pi / 4$ and $v=y-\Im(\alpha) \pi / 2$. Note that

$$
\begin{aligned}
& \cos \left(z-\alpha \frac{\pi}{2}-\frac{\pi}{4}\right)=\cos (u+i v) \quad \text { and } \\
& |\cos (u+i v)|^{2}=\cos ^{2} u+\sinh ^{2} v .
\end{aligned}
$$

Let $R_{k}:=k \pi+\Re(\alpha) \pi / 2+\pi / 4, x_{k}:=R_{k}-\pi / 2$ and $y_{0}:=\Im(\alpha) \pi / 2$. For $1 \leq k \leq N$, consider the two arcs of the circle $|z|=R$ inside the strip $x_{k}-$ $\pi / 2 \leq x \leq x_{k}+\pi / 2$. Suppose that $z=x+i y$ is on an arc for which $x_{k}-\pi / 2 \leq$ $x \leq x_{k}+\pi / 2$ and $\left|y-y_{0}\right| \geq \pi / 4$. Using (20), we have

$$
\left|\cos \left(z-\alpha \frac{\pi}{2}-\frac{\pi}{4}\right)\right|^{2}=\cos ^{2} u+\sinh ^{2} v \geq \sinh ^{2} v \geq\left(\frac{1-e^{-\pi / 2}}{2}\right)^{2} e^{2|v|}
$$

whence

$$
\left|\cos \left(z-\alpha \frac{\pi}{2}-\frac{\pi}{4}\right)\right|>C_{1}(\alpha) e^{R|\sin \theta|} .
$$

If $z=x+i y$ is on an arc for which $x_{k}-\pi / 2 \leq x \leq x_{k}+\pi / 2$ and $\left|y-y_{0}\right|<\pi / 4$, then $\left|x-x_{k}\right|>\sqrt{\pi^{2} / 16-v^{2}}$ (recall that $N$ is taken sufficiently large). But $\left|x-x_{k}\right|=|u-k \pi+\pi / 2| \leq \pi / 2$, and so

$$
\begin{gathered}
\left|\cos \left(z-\alpha \frac{\pi}{2}-\frac{\pi}{4}\right)\right|^{2}=\cos ^{2} u+\sinh ^{2} v=\sin ^{2}\left(u-k \pi+\frac{\pi}{2}\right)+\sinh ^{2} v \\
=\sin ^{2}\left|x-x_{k}\right|+\sinh ^{2} v>\sin ^{2}\left(\sqrt{\frac{\pi^{2}}{16}-v^{2}}\right)+\sinh ^{2} v=: A(v) .
\end{gathered}
$$

The function $e^{-2|v|} A(v)$ is a positive continuous function in the closed interval $[-\pi / 4, \pi / 4]$. Thus, it has a positive minimum in that interval, say $D$. It follows that

$$
\left|\cos \left(z-\alpha \frac{\pi}{2}-\frac{\pi}{4}\right)\right|>\sqrt{D} e^{|v|}>C_{2}(\alpha) e^{R|\sin \theta|}
$$

We shall also need the following result, which is a simple variant of [3, Lemmas 1 and 2].

Lemma 2. Let $f \in B_{\sigma}$ such that $f(x)=O\left(|x|^{-\delta}\right), x \rightarrow \pm \infty$. If $R \rightarrow \infty$, then we have, uniformly for $|\theta| \leq \pi$,

$$
f\left(R e^{i \theta}\right)=O\left(\frac{e^{\sigma R|\sin \theta|}}{R^{\delta}}\right) .
$$

Moreover,

$$
f^{\prime}(x)=O\left(|x|^{-\delta}\right), \quad x \rightarrow \pm \infty .
$$

Lemma 3. Let $\Re(\alpha)>-1$. For all $f \in B_{2}$ such that $f(x)=O\left(|x|^{-\delta}\right), x \rightarrow$ $\pm \infty$, with $\delta>2 \Re(\alpha)+1$, we have

$$
f(x)=\sum_{\substack{k=-\infty \\ k \neq 0}}^{\infty}\left[A_{k}(x) f\left(j_{k}\right)+B_{k}(x) f^{\prime}\left(j_{k}\right)\right]
$$


where

$$
A_{k}(x):=\left(1+\frac{2 \alpha+1}{j_{k}}\left(x-j_{k}\right)\right)\left(\frac{j_{k}^{\alpha} J_{\alpha}(x)}{x^{\alpha} J_{\alpha}^{\prime}\left(j_{k}\right)\left(x-j_{k}\right)}\right)^{2}
$$

and

$$
B_{k}(x):=\left(x-j_{k}\right)\left(\frac{j_{k}^{\alpha} J_{\alpha}(x)}{x^{\alpha} J_{\alpha}^{\prime}\left(j_{k}\right)\left(x-j_{k}\right)}\right)^{2} .
$$

Proof. Consider the integral

$$
I_{N}(x):=\frac{1}{2 \pi i} \oint_{C_{N}} \frac{z^{2 \alpha} f(z)}{(z-x) J_{\alpha}^{2}(z)} d z,
$$

where $C_{N}$ is the circle $|z|=R:=N \pi+\Re(\alpha) \pi / 2+\pi / 4$. As remarked before, the function $J_{\alpha}(z) / z^{\alpha}$ is entire. Using Lemmas 1 and 2, we have, for large $R$,

$$
\left|I_{N}(x)\right| \leq \frac{1}{2 \pi} \int_{-\pi}^{\pi} \frac{R^{2 \Re(\alpha)+1}\left|f\left(R e^{i \theta}\right)\right|}{\left|R e^{i \theta}-x\right|\left|J_{\alpha}\left(R e^{i \theta}\right)\right|^{2}} d \theta \leq K(\alpha, x) R^{2 \Re(\alpha)+1-\delta} .
$$

Thus,

$$
\lim _{N \rightarrow \infty} I_{N}(x)=0 .
$$

On the other hand, using the residue theorem, we have

$$
I_{N}(x)=\operatorname{Res}(z=x)+\sum_{\left|j_{k}\right|<R} \operatorname{Res}\left(z=j_{k}\right),
$$

and the result follows after a few calculations by letting $N \rightarrow \infty$.

The following properties of Bessel functions will be used, in conjunction with Lemma 3, to prove (8).

Lemma 4. Let $\Re(\alpha)>-1$. For any zero $j_{k}$ of $J_{\alpha}(z) / z^{\alpha}$, we have

$$
\int_{0}^{\infty} x \frac{J_{\alpha}^{2}(x)}{x^{2}-j_{k}^{2}} d x=0
$$

and

$$
\int_{0}^{\infty} x J_{\alpha}^{2}(x)\left(\frac{1}{\left(x-j_{k}\right)^{2}}+\frac{1}{\left(x+j_{k}\right)^{2}}\right) d x=2 .
$$

Proof. Let $R>\varepsilon>0$. First we prove (31). We consider the curve $\Gamma$ which is the union of the two intervals $[-R,-\varepsilon],[\varepsilon, R]$ and the two semicircles $C_{\varepsilon}$, $C_{R}$, where $C_{\delta}:=\{z:|z|=\delta$ and $\Im(z) \geq 0\}$. We have

$$
\oint_{\Gamma} \frac{z J_{\alpha}(z) H_{\alpha}^{(1)}(z)}{z^{2}-j_{k}^{2}} d z=0
$$

where

$$
H_{\alpha}^{(1)}(z)=\frac{J_{-\alpha}(z)-e^{-\alpha \pi i} J_{\alpha}(z)}{i \sin \alpha \pi}
$$

is a Bessel function of the third kind, with the usual interpretation when $\alpha$ is an integer. From (33), we obtain

$$
\int_{-R}^{-\varepsilon} F(x) d x+\int_{C_{\varepsilon}} F(z) d z+\int_{\varepsilon}^{R} F(x) d x+\int_{C_{R}} F(z) d z=0,
$$


where

$$
F(z):=\frac{z J_{\alpha}(z) H_{\alpha}^{(1)}(z)}{z^{2}-j_{k}^{2}}
$$

On $C_{\varepsilon}$ we have $|z|=\varepsilon ;$ since $\lim _{z \rightarrow 0} J_{\alpha}(z) / z^{\alpha}=1 /\left(2^{\alpha} \Gamma(\alpha+1)\right)$, we obtain, if $\alpha$ is not an integer,

$$
|F(z)| \leq K_{1}(\alpha) \varepsilon+K_{2}(\alpha) \varepsilon^{1+2 \Re(\alpha)} .
$$

When $\alpha=n$ is an integer, we need to use, in addition, a representation formula for $H_{\alpha}^{(1)}(z)$; we have $H_{n}^{(1)}(z)=J_{n}(z)+i Y_{n}(z)$, with

$$
\begin{aligned}
\pi Y_{n}(z)= & 2\left(\gamma+\ln \frac{z}{2}\right) J_{n}(z)-\sum_{\kappa=0}^{n-1} \frac{(n-\kappa-1) !}{\kappa !}\left(\frac{z}{2}\right)^{2 \kappa-n} \\
& -\sum_{\kappa=0}^{\infty} \frac{(-1)^{\kappa}}{\kappa !(n+\kappa) !}\left(\frac{z}{2}\right)^{2 \kappa+n} \\
& \quad \times\left(\frac{1}{1}+\frac{1}{2}+\cdots+\frac{1}{\kappa}+\frac{1}{1}+\frac{1}{2}+\cdots+\frac{1}{n+\kappa}\right) .
\end{aligned}
$$

We obtain

It follows that

$$
|F(z)| \leq K_{3}(\alpha) \varepsilon+K_{4}(\alpha) \varepsilon^{1+2 \Re(\alpha)}|\ln \varepsilon|
$$

$$
\lim _{\varepsilon \rightarrow 0} \int_{C_{\varepsilon}} F(z) d z=0 \text { for } \Re(\alpha)>-1 .
$$

On $C_{R}$ we use the asymptotic formula (18) and

$$
H_{\alpha}^{(1)}(z)=\sqrt{\frac{2}{\pi z}} e^{i\left(z-\alpha \frac{\pi}{2}-\frac{\pi}{4}\right)}\left(1+O\left(|z|^{-1}\right)\right)
$$

to obtain

$$
|F(z)| \leq \frac{K_{5}(\alpha)}{R^{2}}
$$

Thus,

$$
\lim _{R \rightarrow \infty} \int_{C_{R}} F(z) d z=0 .
$$

Also, using $H_{\alpha}^{(1)}(x)-e^{\alpha \pi i} H_{\alpha}^{(1)}(-x)=2 J_{\alpha}(x)$, we get

$$
\int_{-R}^{-\varepsilon} F(x) d x+\int_{\varepsilon}^{R} F(x) d x=\int_{\varepsilon}^{R}(F(x)+F(-x)) d x=2 \int_{\varepsilon}^{R} x \frac{J_{\alpha}^{2}(x)}{x^{2}-j_{k}^{2}} d x
$$

and so (31) follows from (34), (36), and (37).

Now we prove (32). We need to distinguish two cases according as $j_{k}$ is real or not. When $j_{k}$ is not real, we consider the contour $\Gamma$ defined in the proof of (31). Only one zero, $j_{k}$ or $-j_{k}$, is inside $\Gamma$. Thus, by the residue theorem,

$$
\begin{aligned}
& \frac{1}{2 \pi i} \oint_{\Gamma} z J_{\alpha}(z) H_{\alpha}^{(1)}(z)\left(\frac{1}{\left(z-j_{k}\right)^{2}}+\frac{1}{\left(z+j_{k}\right)^{2}}\right) d z \\
& \quad=j_{k} J_{\alpha}^{\prime}\left(j_{k}\right) H_{\alpha}^{(1)}\left(j_{k}\right)=j_{k} J_{\alpha}^{\prime}\left(j_{k}\right) \frac{J_{-\alpha}\left(j_{k}\right)}{i \sin \alpha \pi}=\frac{2}{\pi i}
\end{aligned}
$$


since

$$
J_{\alpha}^{\prime}(z) J_{-\alpha}(z)-J_{-\alpha}^{\prime}(z) J_{\alpha}(z)=\frac{2 \sin \alpha \pi}{\pi z} .
$$

The integrals along $C_{\varepsilon}$ and $C_{R}$ tend to zero, as $\varepsilon \rightarrow 0$ and $R \rightarrow \infty$; the argument is analogous to the one used to obtain (36) and (37). Hence, using a decomposition of the form (34), we get

$\frac{1}{2 \pi i} \int_{0}^{\infty} x\left(J_{\alpha}(x) H_{\alpha}^{(1)}(x)-J_{\alpha}(-x) H_{\alpha}^{(1)}(-x)\right)\left(\frac{1}{\left(x-j_{k}\right)^{2}}+\frac{1}{\left(x+j_{k}\right)^{2}}\right) d x=\frac{2}{\pi i}$,

which readily gives us $(32)$.

Now we suppose that $j_{k}$ is real. Consider the curve $\Gamma_{1}$ obtained from $\Gamma$ by replacing the interval $\left[-j_{k}-\delta,-j_{k}+\delta\right]$ by the semicircle $C_{1, \delta}=\left\{z:\left|z+j_{k}\right|=\right.$ $\delta$ and $\Im(z) \geq 0$, where $\delta$ is sufficiently small. We have

$$
\oint_{\Gamma_{1}} F_{1}(z) d z=0, \quad \text { where } F_{1}(z):=\frac{z J_{\alpha}(z) H_{\alpha}^{(1)}(z)}{\left(z+j_{k}\right)^{2}} .
$$

It follows that

(40) $\int_{-\infty}^{-j_{k}-\delta} F_{1}(x) d x+\int_{C_{1, \delta}} F_{1}(z) d z+\int_{-j_{k}+\delta}^{0} F_{1}(x) d x+\int_{0}^{\infty} F_{1}(x) d x=0$.

Similarly,

$$
\int_{-\infty}^{0} F_{2}(x) d x+\int_{0}^{j_{k}-\delta} F_{2}(x) d x+\int_{C_{2, \delta}} F_{2}(z) d z+\int_{j_{k}+\delta}^{\infty} F_{2}(x) d x=0,
$$

where

$$
F_{2}(z):=\frac{z J_{\alpha}(z) H_{\alpha}^{(1)}(z)}{\left(z-j_{k}\right)^{2}} \quad \text { and } \quad C_{2, \delta}=\left\{z:\left|z-j_{k}\right|=\delta \text { and } \Im(z) \geq 0\right\} \text {. }
$$

We add (40) and (41) to obtain

$$
\begin{aligned}
0= & \int_{0}^{j_{k}-\delta}\left(F_{1}(-x)+F_{2}(x)\right) d x+\int_{j_{k}+\delta}^{\infty}\left(F_{1}(-x)+F_{2}(x)\right) d x \\
& +\int_{0}^{\infty}\left(F_{1}(x)+F_{2}(-x)\right) d x+\int_{C_{1, \delta}} F_{1}(z) d z+\int_{C_{2, \delta}} F_{2}(z) d z,
\end{aligned}
$$

whence

$$
\begin{aligned}
0= & 2 \int_{0}^{j_{k}-\delta} x \frac{J_{\alpha}^{2}(x)}{\left(x-j_{k}\right)^{2}} d x+2 \int_{j_{k}+\delta}^{\infty} x \frac{J_{\alpha}^{2}(x)}{\left(x-j_{k}\right)^{2}} d x \\
& +2 \int_{0}^{\infty} x \frac{J_{\alpha}^{2}(x)}{\left(x+j_{k}\right)^{2}} d x+\int_{C_{1, \delta}} F_{1}(z) d z+\int_{C_{2, \delta}} F_{2}(z) d z
\end{aligned}
$$

Taking the limit as $\delta \rightarrow 0$, we get

$$
\begin{aligned}
0= & 2 \int_{0}^{\infty} x J_{\alpha}^{2}(x)\left(\frac{1}{\left(x-j_{k}\right)^{2}}+\frac{1}{\left(x+j_{k}\right)^{2}}\right) d x \\
& +\lim _{\delta \rightarrow 0}\left(\int_{C_{1, \delta}} F_{1}(z) d z+\int_{C_{2, \delta}} F_{2}(z) d z\right) .
\end{aligned}
$$


It remains to evaluate the last limit. We have, if $\alpha$ is not an integer,

$$
\begin{aligned}
\lim _{\delta \rightarrow 0} & \left(\int_{C_{1, \delta}} F_{1}(z) d z\right) \\
& =i \lim _{\delta \rightarrow 0} \int_{\pi}^{0}\left(-j_{k}+\delta e^{i \theta}\right) \frac{J_{\alpha}\left(-j_{k}+\delta e^{i \theta}\right)}{\delta e^{i \theta}} H_{\alpha}^{(1)}\left(-j_{k}+\delta e^{i \theta}\right) d \theta \\
& =i \pi j_{k} J_{\alpha}^{\prime}\left(-j_{k}\right) H_{\alpha}^{(1)}\left(-j_{k}\right)=\pi j_{k} \frac{J_{\alpha}^{\prime}\left(-j_{k}\right) J_{-\alpha}\left(-j_{k}\right)}{\sin \alpha \pi} \\
& =-\pi j_{k} \frac{J_{\alpha}^{\prime}\left(j_{k}\right) J_{-\alpha}\left(j_{k}\right)}{\sin \alpha \pi}
\end{aligned}
$$

and

$$
\lim _{\delta \rightarrow 0}\left(\int_{C_{2, \delta}} F_{2}(z) d z\right)=-\pi j_{k} \frac{J_{\alpha}^{\prime}\left(j_{k}\right) J_{-\alpha}\left(j_{k}\right)}{\sin \alpha \pi} .
$$

Using (38), we readily obtain

$$
\lim _{\delta \rightarrow 0}\left(\int_{C_{1, \delta}} F_{1}(z) d z+\int_{C_{2, \delta}} F_{2}(z) d z\right)=-4,
$$

and (32) follows from (44). When $\alpha$ is an integer, the function $H_{\alpha}^{(1)}(z)$ has to be replaced by its limit.

\section{Proofs of THE THEOREMS}

We may obviously suppose $\tau=1$.

Proof of Theorem 1. Using Lemma 3, we have

$$
f(x)+f(-x)=\sum_{\substack{k=-\infty \\ k \neq 0}}^{\infty}\left(A_{k}(x)+A_{k}(-x)\right) f\left(j_{k}\right)+\left(B_{k}(x)+B_{k}(-x)\right) f^{\prime}\left(j_{k}\right),
$$

that is,

$$
\begin{aligned}
f(x)+f(-x)= & \sum_{\substack{k=-\infty \\
k \neq 0}}^{\infty} \frac{j_{k}^{2 \alpha}}{\left(J_{\alpha}^{\prime}\left(j_{k}\right)\right)^{2}} \frac{J_{\alpha}^{2}(x)}{x^{2 \alpha}}\left(\frac{1}{\left(x-j_{k}\right)^{2}}+\frac{1}{\left(x+j_{k}\right)^{2}}\right) f\left(j_{k}\right) \\
& +\sum_{\substack{k=-\infty \\
k \neq 0}}^{\infty} 2(2 \alpha+1) \frac{j_{k}^{2 \alpha}}{\left(J_{\alpha}^{\prime}\left(j_{k}\right)\right)^{2}} \frac{J_{\alpha}^{2}(x)}{x^{2 \alpha}\left(x^{2}-j_{k}^{2}\right)} f\left(j_{k}\right) \\
& +\sum_{\substack{k=-\infty \\
k \neq 0}}^{\infty} 2 \frac{j_{k}^{2 \alpha+1}}{\left(J_{\alpha}^{\prime}\left(j_{k}\right)\right)^{2}} \frac{J_{\alpha}^{2}(x)}{x^{2 \alpha}\left(x^{2}-j_{k}^{2}\right)} f^{\prime}\left(j_{k}\right) .
\end{aligned}
$$

Multiplying by $x^{2 \alpha+1}$ and integrating, we obtain formally the formula (8) with the help of Lemma 4. Hence, it remains to justify the interchanges of the order of integration and summation. First observe that, in order to prove formula (8), we may assume

$$
f(x)=O\left(|x|^{-\delta}\right), \quad x \rightarrow \pm \infty, \delta>2 \Re(\alpha)+6 .
$$


Indeed, if $f(x)=O\left(|x|^{-\delta}\right), x \rightarrow \pm \infty$, with only $\delta>2 \Re(\alpha)+2$, then the functions

$$
g_{\varepsilon}(z):=\left(\frac{\sin \varepsilon z}{\varepsilon z}\right)^{4} f(z), \quad \varepsilon>0, \quad f \in B_{2 \tau},
$$

satisfy the hypothesis of Theorem 1 with $\tau$ replaced by $\tau+2 \varepsilon$ and $\delta>2 \Re(\alpha)+2$ replaced by $\delta>2 \Re(\alpha)+6$. Thus,

$$
\begin{aligned}
\int_{0}^{\infty} & x^{2 \alpha+1}\left(g_{\varepsilon}(x)+g_{\varepsilon}(-x)\right) d x \\
& =\frac{2}{(\tau+2 \varepsilon)^{2 \alpha+2}} \sum_{k=1}^{\infty} \frac{j_{k}^{2 \alpha}}{\left(J_{\alpha}^{\prime}\left(j_{k}\right)\right)^{2}}\left(g_{\varepsilon}\left(\frac{j_{k}}{\tau+2 \varepsilon}\right)+g_{\varepsilon}\left(-\frac{j_{k}}{\tau+2 \varepsilon}\right)\right) .
\end{aligned}
$$

The passage to the limit as $\varepsilon \rightarrow 0$, in (47), is easily justified using

$$
\left|\frac{\sin \varepsilon x}{\varepsilon x}\right| \leq 1 \quad \text { and } \quad f\left( \pm \frac{j_{k}}{\tau+2 \varepsilon}\right)=O\left(\frac{(\tau+2 \varepsilon)^{\delta}}{\left|j_{k}\right|^{\delta}}\right) .
$$

Now we must prove, in particular, that

$$
\begin{aligned}
\int_{0}^{\infty} & \left(\sum_{\substack{k=-\infty \\
k \neq 0}}^{\infty} 2 \frac{j_{k}^{2 \alpha+1}}{\left(J_{\alpha}^{\prime}\left(j_{k}\right)\right)^{2}} \frac{x J_{\alpha}^{2}(x)}{x^{2}-j_{k}^{2}} f^{\prime}\left(j_{k}\right)\right) d x \\
& =\sum_{\substack{k=-\infty \\
k \neq 0}}^{\infty} 2 \frac{j_{k}^{2 \alpha+1}}{\left(J_{\alpha}^{\prime}\left(j_{k}\right)\right)^{2}} f^{\prime}\left(j_{k}\right) \int_{0}^{\infty} \frac{x J_{\alpha}^{2}(x)}{x^{2}-j_{k}^{2}} d x
\end{aligned}
$$

The other interchanges could be proved, with a slight simplification, as is done in the following argument. In view of Lebesgue's dominated convergence theorem, it suffices to show that there exists an integrable function $G(x)$ such that

$$
\sum_{\substack{k=-\infty \\ k \neq 0}}^{\infty} \lambda_{k}(x) \leq G(x), \quad \text { where } \lambda_{k}(x):=\left|\frac{j_{k}^{2 \alpha+1}}{\left(J_{\alpha}^{\prime}\left(j_{k}\right)\right)^{2}} \frac{x J_{\alpha}^{2}(x)}{x^{2}-j_{k}^{2}} f^{\prime}\left(j_{k}\right)\right| \text {. }
$$

For small values of $x$, we use $J_{\alpha}(x)=O\left(|x|^{\Re(\alpha)}\right)$ as $x \rightarrow 0,(24)$ and the hypothesis (46) to obtain

$$
\sum_{\substack{k=-\infty \\ k \neq 0}}^{\infty} \lambda_{k}(x) \leq K_{1}(\alpha) x^{2 \Re(\alpha)+1}
$$

For large values of $x$, we write

$$
\sum_{\substack{k=-\infty \\ k \neq 0}}^{\infty} \lambda_{k}(x)=I_{1}+I_{2}+I_{3}
$$

where

$$
I_{1}:=\sum_{\left|j_{k}\right|<\frac{x}{2}} \lambda_{k}(x), \quad I_{2}:=\sum_{\frac{x}{2} \leq\left|j_{k}\right| \leq \frac{3 x}{2}} \lambda_{k}(x) \text { and } I_{3}:=\sum_{\frac{3 x}{2}<\left|j_{k}\right|} \lambda_{k}(x) .
$$


We have, for $x / 2 \leq\left|j_{k}\right| \leq 3 x / 2$,

$$
\left|\frac{J_{\alpha}^{2}(x)}{x^{2}-j_{k}^{2}}\right| \leq C_{1}(\alpha)
$$

which follows from

$$
J_{\alpha}(x)=\int_{ \pm j_{k}}^{x} J_{\alpha}^{\prime}(u) d u
$$

and the asymptotic formula

$$
J_{\alpha}^{\prime}(u)=-\sqrt{\frac{2}{\pi u}} \sin \left(u-\alpha \frac{\pi}{2}-\frac{\pi}{4}\right)\left(1+O\left(|u|^{-1}\right)\right) .
$$

Thus, using also (46) and (24), we obtain

$$
I_{2} \leq \frac{C_{1}(\alpha)}{x^{2}} \sum_{\frac{x}{2} \leq\left|j_{k}\right| \leq \frac{3 x}{2}}\left|j_{k}\right|^{2 \Re(\alpha)+2-\delta} x^{3} \leq 8 \frac{C_{1}(\alpha)}{x^{2}} \sum_{\substack{k=-\infty \\ k \neq 0}}^{\infty}\left|j_{k}\right|^{2 \Re(\alpha)+5-\delta} \leq \frac{C_{2}(\alpha)}{x^{2}} .
$$

In order to obtain an upper bound for $I_{1}$ and $I_{3}$, we use (46), (24) and the above asymptotic formula for $J_{\alpha}^{\prime}$. We get

$$
I_{1} \leq C_{3}(\alpha) \sum_{\left|j_{k}\right|<\frac{x}{2}} \frac{\left|j_{k}\right|^{2 \Re(\alpha)+2-\delta}}{x^{2}-\left|j_{k}\right|^{2}} \leq \frac{4}{3} \frac{C_{3}(\alpha)}{x^{2}} \sum_{\substack{k=-\infty \\ k \neq 0}}^{\infty}\left|j_{k}\right|^{2 \Re(\alpha)+2-\delta} \leq \frac{C_{4}(\alpha)}{x^{2}}
$$

and

$$
I_{3} \leq C_{5}(\alpha) \sum_{\frac{3 x}{2}<\left|j_{k}\right|} \frac{\left|j_{k}\right|^{2 \Re(\alpha)+2-\delta}}{\left|j_{k}\right|^{2}-x^{2}} \leq \frac{4}{5} \frac{C_{5}(\alpha)}{x^{2}} \sum_{\substack{k=-\infty \\ k \neq 0}}^{\infty}\left|j_{k}\right|^{2 \Re(\alpha)+2-\delta} \leq \frac{C_{6}(\alpha)}{x^{2}} .
$$

From (50), we deduce that

$$
\sum_{\substack{k=-\infty \\ k \neq 0}}^{\infty} \lambda_{k}(x) \leq \frac{K_{2}(\alpha)}{x^{2}}
$$

for large values of $x$.

Proof of Theorem 2. Consider two quadrature formulas of the form (15) with nodal functions $\omega_{1}(x)$ and $\omega_{2}(x)$, respectively. Suppose $\omega_{1}(x) \not \equiv \omega_{2}(x)$, and let $h(x):=\omega_{1}(x)-\omega_{2}(x)$. The function $h(x) \omega_{1}(x)$ is in $B_{2}$ and vanishes at the nodes $x_{k}$ (including $k=m$ ) associated with the quadrature formula with $\omega_{1}(x)$ as nodal function. Moreover, $h(x) \omega_{1}(x)=O\left(|x|^{-2 \alpha-3}\right), x \rightarrow \pm \infty$. Therefore,

$$
\int_{0}^{\infty} x^{2 \alpha+1}\left(h(x) \omega_{1}(x)+h(-x) \omega_{1}(-x)\right) d x=0 .
$$

Since $h(x) \not \equiv 0$, we have

$$
\int_{0}^{\infty} x^{2 \alpha+1}\left(h^{2}(x)+h^{2}(-x)\right) d x>0,
$$


and so, using (51),

$$
\int_{0}^{\infty} x^{2 \alpha+1}\left(\omega_{2}^{2}(x)+\omega_{2}^{2}(-x)\right) d x>\int_{0}^{\infty} x^{2 \alpha+1}\left(\omega_{1}^{2}(x)+\omega_{1}^{2}(-x)\right) d x
$$

Considering, instead of $h(x) \omega_{1}(x)$, the function $h(x) \omega_{2}(x)$, we are led similarly to

$$
\int_{0}^{\infty} x^{2 \alpha+1}\left(\omega_{1}^{2}(x)+\omega_{1}^{2}(-x)\right) d x>\int_{0}^{\infty} x^{2 \alpha+1}\left(\omega_{2}^{2}(x)+\omega_{2}^{2}(-x)\right) d x
$$

which contradicts $(52)$. Thus $\omega_{1}(x) \equiv \omega_{2}(x)$.

Since $\omega_{e}(x)$ is the nodal function associated with (8), we deduce, by the same argument, that

$$
\int_{0}^{\infty} x^{2 \alpha+1}\left(\omega^{2}(x)+\omega^{2}(-x)\right) d x>\int_{0}^{\infty} x^{2 \alpha+1}\left(\omega_{e}^{2}(x)+\omega_{e}^{2}(-x)\right) d x,
$$

for all nodal functions $\omega(x) \not \equiv \omega_{e}(x)$.

\section{REMARKS AND EXAMPLES}

5.1. Theorem 1 does not remain valid for the class $B_{\sigma}$ if $\sigma>2 \tau$. Any function of the form

$$
f_{*}(z)=\frac{J_{\alpha}(\tau z)}{z^{\alpha}} \frac{J_{\bar{\alpha}}(\tau z)}{z^{\bar{\alpha}}}\left(\frac{J_{\eta / 2}\left(\frac{\varepsilon z}{2}\right)}{z^{\eta / 2}}\right)^{2}, \quad \eta, \varepsilon>0,
$$

is a counterexample. The function $f_{*}$ is in the class $B_{2 \tau+\varepsilon}$ and $f_{*}(x)=$ $O\left(|x|^{-\delta}\right), x \rightarrow \pm \infty$, with $\delta=2 \Re(\alpha)+2+\eta$. The summation in the righthand member of $(8)$ is clearly zero, but the integral of the left-hand member is positive, since

$$
f_{*}(x)=\left|\frac{J_{\alpha}(\tau x)}{x^{\alpha}}\right|^{2}\left(\frac{J_{\eta / 2}(\varepsilon x / 2)}{x^{\eta / 2}}\right)^{2} .
$$

5.2. The hypothesis $\delta>2 \Re(\alpha)+2$ cannot be relaxed in Theorem 1. Consider the function

$$
f_{*}(z)=\frac{J_{\alpha}(\tau z) J_{\alpha+1}(\tau z)}{z^{2 \alpha+1}}
$$

This function is in the class $B_{2 \tau}$ and $f_{*}(x)=O\left(|x|^{-\delta}\right), x \rightarrow \pm \infty$, with $\delta=2 \Re(\alpha)+2$. The summation in the right-hand member of (8) is clearly zero but, for $\Re(\alpha)>-1$,

$$
\int_{0}^{\infty} x^{2 \alpha+1}\left(f_{*}(x)+f_{*}(-x)\right) d x=2 \int_{0}^{\infty} J_{\alpha}(\tau x) J_{\alpha+1}(\tau x) d x=\frac{1}{\tau}
$$

5.3. When $\alpha=m+1 / 2$, where $m$ is a nonnegative integer, the functions $J_{\alpha}(z)$ take the following explicit form:

$$
J_{m+1 / 2}(z)=\sqrt{\frac{2 z}{\pi}} z^{m}\left(-\frac{1}{z} \frac{d}{d z}\right)^{m}\left(\frac{\sin z}{z}\right) .
$$

In the case $m=1$, formula (8) becomes

$$
\int_{-\infty}^{\infty} x^{4} f(x) d x=\frac{32 \pi}{\tau^{5}} \sum_{k=-\infty}^{\infty} r_{k}^{2}\left(1+r_{k}^{2}\right) f\left(\frac{r_{k}}{\tau}\right),
$$

where $f \in B_{2 \tau}$ satisfies $f(x)=O\left(|x|^{-\delta}\right), x \rightarrow \pm \infty$, with $\delta>5$ and $r_{k}$, $-\infty<k<\infty$, are the roots of the equation $\tan x=x$. 
5.4. Let

$$
\varphi_{m}(z):=\frac{\sqrt{2} j_{m} J_{\alpha}(z)}{z^{\alpha}\left(z^{2}-j_{m}^{2}\right)} .
$$

If we take, in Theorem 1, $\tau=1$ and $f(z)=\varphi_{m}(z) \varphi_{l}(z)$, then we obtain

$$
\int_{0}^{\infty} x^{2 \alpha+1} \varphi_{m}(x) \varphi_{l}(x) d x= \begin{cases}0, & l \neq m, \\ 1, & l=m .\end{cases}
$$

In other words, the family $\left\{\varphi_{m}: m= \pm 1, \pm 2, \ldots\right\}$ is orthonormal on $[0, \infty)$ with weight function $x^{2 \alpha+1}$.

\section{BIBLIOGRAPHY}

1. R. P. Boas, Jr., Summation formulas and band-limited signals, Tôhoku Math. J. 24 (1972), 121-125.

2. C. Frappier et Q. I. Rahman, Une formule de quadrature pour les fonctions entières de type exponentiel, Ann. Sci. Math. Québec 10 (1986), 17-26.

3. R. Gervais, Q. I. Rahman and G. Schmeisser, Representation and approximation of functions via (0, 2)-interpolation, J. Approx. Theory 50 (1987), 89-110.

4. G. H. Hardy, Notes on special systems of orthogonal functions IV: the orthogonal functions of Whittaker's cardinal series, Proc. Cambridge Philos. Soc. 37 (1941), 331-348.

5. Q. I. Rahman and G. Schmeisser, An analogue of Turán's quadrature formula, Constr. Theory of Functions, Sofia, 1988, pp. 405-412.

6. G. Szegö, Orthogonal polynomials, 4th ed., Amer. Math. Soc. Colloq. Publ., Vol. 23, Providence, R.I., 1975.

7. G. N. Watson, A treatise on the theory of Bessel functions, 2nd ed., Cambridge Univ. Press, Cambridge, 1952.

École Polytechnique, Departement de Mathematiques Appliquées, CP 6079, Succ. A, Montréal (PQ), Canada, H3C 3A7 